\title{
Positive feedback
}

DOI:

10.1038/nrc2024

\section{URLs}

p53

http://www.ncbi.nlm.nih.gov/

entrez/query.fcgi?db=gene\&c

$\mathrm{md}=$ Retrieve\&dopt=full_

report\&list_uids=7157

LATS2

http://www.ncbi.nlm.nih.gov/ entrez/query.fcgi?db=gene\&c $\mathrm{md}=$ Retrieve\&dopt=full reportElist_uids $=26524$

MDM2

http://www.ncbi.nlm.nih.gov/ entrez/query.fcgi?db=gene\&c $\mathrm{md}=$ Retrieve\&dopt=full reportElist_uids $=4193$
The mechanisms that drive postmitotic checkpoint responses after damage to the mitotic spindle and centrosomes are poorly understood. Moshe Oren and colleagues now report that the tumour suppressor LATS2 interacts with MDM2, a key negative regulator of the tumour suppressor $\mathrm{p} 53$, to promote $\mathrm{p} 53$ activation in cells that have mitoticspindle damage.

Having established that LATS2 binds to MDM2 when cells are treated with the spindle-damaging agent nocodazole, the authors investigated the effects of this interaction. Although the overexpression of MDM2 promoted $\mathrm{p} 53$ degradation in mammalian cell lines as expected, the co-expression of LATS2 inhibited MDM2 E3 ubiquitin ligase activity, and consequently attenuated the degradation of p53. As LATS2 is localized with the centrosome during the cell cycle, the authors reasoned that the LATS2-MDM2 interaction might occur specifically when centrosome function was disrupted, and the treatment of mammalian cells with nocodazole did increase the interaction between LATS2 and MDM2. U2OS osteosarcoma cells were transfected with LATS2, and the expression and localization of LATS2,
MDM2 and p53 was examined over time following treatment with nocodazole. Within an hour or so of nocodazole treatment LATS2 began to translocate from the centrosome to the nucleoplasm, and p53 accumulation increased in parallel with this. MDM2 staining remained high and nuclear over the 20 hour observation period. By 20 hours many cells showed mitotic arrest.

As the transcription of LATS2 has been reported to be upregulated by $\mathrm{p} 53$, the authors used U2OS cells wild-type for TP53 or with stably knocked down TP53 to look at the effects of nocodazole treatment on LATS2 mRNA levels. LATS2 levels increased rapidly only in cells with wild-type p53 after treatment with nocodazole. The induction of LATS2 was dependent on cell-cycle position at the time of nocodazole treatment, so ruling out a generalized stress response. So, there is a positive-feedback loop between p53 and LATS2.

What is the functional outcome of the induction of LATS2? Oren and colleagues showed that the abrogation of LATS2 in U2OS cells by stable knockdown promoted the accumulation of polyploid cells after exposure to nocodazole, and this is prevented if p53 is activated directly by treat- ment with the drug Nutlin-3.

The LATS2-MDM2-p53 axis constitutes a new positive-feedback checkpoint pathway crucial for maintaining normal chromosome number. The authors suggest that in genomically unstable cancer cells, LATS2 expression might be suboptimal and rate limiting, enabling the cells to slip into polyploidy even if they have wild-type p53.

Ezzie Hutchinson

ORIGINAL RESEARCH PAPER Aylon, Y, et al. A positive feedback loop between the $\mathrm{p} 53$ and Lats2 tumour suppressors prevents tetraploidization. Genes Dev. 20, 2687-2700 (2006)

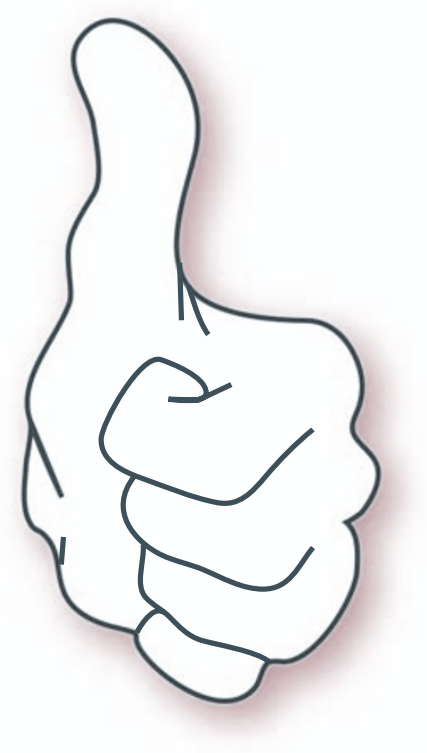

